ARTICLE

Received 11 Jun 2015 | Accepted 29 Jul 2015 | Published 18 Sep $2015 \quad$ DOl: 10.1038/ncomms9242 OPEN

\title{
Molecular helices as electron acceptors in high-performance bulk heterojunction solar cells
}

Yu Zhong 1 , M. Tuan Trinh1, Rongsheng Chen¹,2, Geoffrey E. Purdum³, Petr P. Khlyabich' ${ }^{3}$ Melda Sezen ${ }^{3}$, Seokjoon Oh', Haiming Zhu' ${ }^{1}$, Brandon Fowler', Boyuan Zhang ${ }^{1}$, Wei Wang ${ }^{1}$, Chang-Yong Nam ${ }^{4}$,

Matthew Y. Sfeir ${ }^{4}$, Charles T. Black ${ }^{4}$, Michael L. Steigerwald ${ }^{1}$, Yueh-Lin Loo ${ }^{3}$, Fay Ng${ }^{1}$, X.-Y. Zhu ${ }^{1}$ \& Colin Nuckolls ${ }^{1}$

Despite numerous organic semiconducting materials synthesized for organic photovoltaics in the past decade, fullerenes are widely used as electron acceptors in highly efficient bulk-heterojunction solar cells. None of the non-fullerene bulk heterojunction solar cells have achieved efficiencies as high as fullerene-based solar cells. Design principles for fullerene-free acceptors remain unclear in the field. Here we report examples of helical molecular semiconductors as electron acceptors that are on par with fullerene derivatives in efficient solar cells. We achieved an $8.3 \%$ power conversion efficiency in a solar cell, which is a record high for non-fullerene bulk heterojunctions. Femtosecond transient absorption spectroscopy revealed both electron and hole transfer processes at the donor - acceptor interfaces. Atomic force microscopy reveals a mesh-like network of acceptors with pores that are tens of nanometres in diameter for efficient exciton separation and charge transport. This study describes a new motif for designing highly efficient acceptors for organic solar cells.

\footnotetext{
${ }^{1}$ Department of Chemistry, Columbia University, 3000 Broadway, Havemeyer Hall, MC3130, New York, New York 10027, USA. ${ }^{2}$ School of Chemical Engineering and Technology, Wuhan University of Science and Technology, Wuhan 430081, China. ${ }^{3}$ Department of Chemical and Biological Engineering, Princeton University, Princeton, New Jersey 08544, USA. ${ }^{4}$ Center for Functional Nanomaterials, Brookhaven National Laboratory, Upton, New York, New York 11973, USA. Correspondence and requests for materials should be addressed to F.N. (email: fwn2@columbia.edu) or to X.-Y.Z (email: xz2324@columbia.edu) or to C.N. (email: cn37@columbia.edu).
} 
$\mathrm{n}$ the past decade, the record power conversion efficiency (PCE) of organic bulk heterojunction $(\mathrm{BHJ})$ solar cells has reached over $10 \%$ for single junction cells ${ }^{1,2}$ and more than $12 \%$ for tandem cells ${ }^{3}$, mainly by synthesizing and incorporating new electron donors ${ }^{4,5}$, optimizing film morphology ${ }^{1,6}$, developing interfacial layers ${ }^{7-9}$ and designing new device structures $^{10-12}$. One lagging area is the development of new electron acceptors for organic solar cells. To date, highly efficient $\mathrm{BHJ}$ solar cells almost exclusively use fullerene derivatives, such as $[6,6]$-phenyl- $\mathrm{C}_{61 / 71}$-butyric acid methyl ester $\left(\mathrm{PC}_{61} \mathrm{BM}\right.$ and $\left.\mathrm{PC}_{71} \mathrm{BM}\right)$, as electron acceptors ${ }^{13}$. Theoretical analysis suggests that the superiority of fullerene over non-fullerene electron acceptors is in the charge separation ${ }^{14}$. Recently, several studies, including our own, have reported solution-processed $\mathrm{BHJ}$ devices using non-fullerene acceptors that have achieved PCEs of $6 \%-7 \%$ (refs 15-19). Continued progress will require the design, synthesis and testing of new motifs for electron acceptors.

We herein use two helical $n$-type molecules that differ in their lengths as highly efficient electron acceptors. These fullerene-free BHJ solar cells achieve PCEs of $8.3 \%$, with high short-circuit current density $\left(15.2 \mathrm{~mA} \mathrm{~cm}^{-2}\right)$ and fill factors (FFs) as large as $68 \%$. The film morphology of the mixed donor/acceptor active layer is a mesh-like network of acceptors with pores that are tens of nanometres in diameter. Transient absorption (TA) spectroscopy reveals that excitons generated in both the donor and acceptor phases contribute to the photocurrent by effective hole and electron transfer at the interface between donor and acceptor.

\section{Results}

Molecule design and characterization. The two helices have different lengths and are constructed by fusing either three or four perylene diimide (PDI) units together with a two-carbon bridge (hPDI3 and hPDI4 in Fig. 1a) ${ }^{20}$. Recently, we found that a shorter version of helical molecules was useful in BHJ solar cells ${ }^{16}$. Similar to the shorter one, the longer acceptors explored here also have relatively high electron mobilities (0.04-0.05 $\mathrm{cm}^{2} \mathrm{~V}^{-1} \mathrm{~s}^{-1}$ in thin film transistors), good electronaccepting ability and lowest unoccupied molecular orbital (LUMO) levels (approximately $-3.9 \mathrm{eV}$ ) that are appropriately matched to those of commercially available electron-donating polymers (Fig. 1b) ${ }^{20}$. Similar to prior non-fullerene electron acceptors that have been moderately successful in solar cells $^{15,21,22}$, these molecules possess nonplanar molecular structures due to the steric congestion in the cove areas defined by the junction point between the PDIs. There are several iso-energetic conformations within these oligomers because of the inversion of the helicity at each of these junctions (Fig. 1c, Supplementary Fig. 1 and Supplementary Note 1). Because of the nonplanar structure and the associated conformational dynamics, these molecules do not aggregate strongly in thin films ${ }^{23}$. Both of the molecules absorb light strongly in the wavelength range from 350 to $600 \mathrm{~nm}$ (Fig. 1d) with a maximum molar extinction coefficient of $1.5 \times 10^{5} \mathrm{M}^{-1} \mathrm{~cm}^{-1}$ for hPDI3 and $1.8 \times 10^{5} \mathrm{M}^{-1} \mathrm{~cm}^{-1}$ for hPDI4 (see Supplementary Fig. 2a.). The strong light absorption, the lack of aggregation and the propensity of the isolated linear structure to form networks ${ }^{24}$ indicate that these two molecules have the potential to outperform PDI monomers and dimers in BHJ solar cells.

Solar cell characterization. Our device demonstrations using the compounds hPDI3 and hPDI4 as electron acceptors are based on the commercially available low bandgap semiconducting polymer donors polythieno[3,4-b]-thiophene-co-benzodithiophene (PTB7) ${ }^{4}$ and poly[4,8-bis(5-(2-ethylhexyl)thiophen-2-yl)benzo[1,2-b;4,5- $\left.\mathrm{b}^{\prime}\right]$ dithiophene-2,6-diyl-alt-(4-(2-ethylhexyl)-3-fluorothieno[3,4-b] thiophene)-2-carboxylate-2,6-diyl] (PTB7-Th) ${ }^{5}$ (shown in Fig. 1a). The absorption bands of PTB7 and PTB7-Th are red shifted relative to those of hPDI3 and hPDI4 (Fig. 1d). Films of hPDI3 or hPDI4 blended with PTB7 or PTB7-Th show broad, intense absorptions spanning the wavelength range from 350 to $800 \mathrm{~nm}$ (Supplementary Fig. 2b).

For each combination of blended polymer and helical molecule device active layer, we varied the ratio of donor and acceptor, and optimized the film morphology with the solvent additive diiodooctane $(\mathrm{DIO})^{6}$ (see details about device optimization process in Supplementary Figs 3-6 and Supplementary Tables 1-4). Our devices were fabricated in an inverted configuration, using $\mathrm{ZnO}$ as the electron transport layer and $\mathrm{MoO}_{3}$ as the hole transport layer ${ }^{13}$. For devices based on acceptor hPDI3, we obtain optimal device performance with donor:acceptor mass ratio of $1: 1.5$, with $1 \%$ DIO additive $(\mathrm{v} / \mathrm{v})$. Typical current density - voltage $(J-V)$ curves for PTB7:hPDI3 and PTB7-Th:hPDI3 solar cells are shown in Fig. 2a. The solar cell parameters are listed in Table 1. PTB7-Th:hPDI3 exhibits a larger $J_{\mathrm{sc}}$ of $14.5 \mathrm{~mA} \mathrm{~cm}^{-2}$ as compared with $13.2 \mathrm{~mA} \mathrm{~cm}^{-2}$ for PTB7:hPDI3, owing to the red-shifted absorption of PTB7-Th relative to PTB7. Although the open-circuit voltage $\left(V_{\mathrm{oc}}\right)$ is comparable at $0.77-0.81 \mathrm{eV}$, the FF increases from $63 \%$ for PTB7:hPDI3 to $67 \%$ for PTB7-Th:hPDI3. Overall, devices comprised of PTB7-Th:hPDI3 outperform those that comprise PTB7:hPDI3, exhibiting a maximal PCE of $7.9 \%$ for PTB7-Th:hPDI3 as compared with $6.4 \%$ for PTB7:hPDI3.

For solar cells based on the electron acceptor hPDI4, we achieved optimal device performance with donor-to-acceptor mass ratio of $1: 1$ and $1 \%$ DIO additive $(\mathrm{v} / \mathrm{v})$. Typical current density-voltage curves are shown in Fig. 2b. Optimized devices show similar performance to those based on hPDI3, with PTB7:hPDI4 and PTB7-Th:hPDI4 solar cells reaching maximum PCE of $6.5 \%$ and $8.3 \%$, respectively (device characteristics summarized in Table 1). Device performance of a PTB7Th:hPDI4 solar cell has been independently certified by Newport Corporation, as shown in Table 1 and Supplementary Fig. 7. These device characteristics are similar to those reported in fullerene-based cells (PTB7-Th:PC ${ }_{71} \mathrm{BM}$ ) with the same interfacial layers $\left(\mathrm{ZnO} \text { and } \mathrm{MoO}_{3}\right)^{2,5,25,26}$ and represent record highs for non-fullerene $\mathrm{BHJ}$, with the highest non-fullerene $\mathrm{BHJ}$ having a PCE of $6.8 \%$ (refs 15-18). These molecules are the first example of electron acceptors that are on par with fullerene derivatives in efficient $\mathrm{BHJ}$ solar cells with a PCE $>8 \%$ and provide a new route to exploring improvements to $\mathrm{BHJ}$ device performance.

Previously reported solar cells with non-fullerene acceptors typically have $J_{\mathrm{sc}}<14 \mathrm{~mA} \mathrm{~cm}^{-2}$ and FF values below $60 \%$ (refs 15-18), which may be indicators of sub-optimal chargecarrier collection. The improved $J_{\mathrm{sc}}$ and FF values in devices containing helical PDIs may be due to the efficient exciton generation, separation and carrier transport. Plots of the photocurrent density $\left(J_{\mathrm{ph}}\right)$ versus effective voltage $\left(V_{\text {eff }}\right)$ yield information about exciton generation rates and the charge collection probabilities $P(E, T)$ at $J_{\text {sc }}$ conditions (Supplementary Fig. 8). Here, $V_{\text {eff }}$ is defined as $V_{\text {eff }}=V_{0}-V_{\mathrm{a}}$, where $V_{0}$ is the voltage where $J_{\mathrm{ph}}$ equals zero and $V_{\mathrm{a}}$ is the applied bias voltage. For both hPDI3-based and hPDI4-based devices, $J_{\mathrm{ph}}$ reaches the saturation current density at a relatively low $V_{\text {eff }}(1.8 \mathrm{~V})$. The PTB7-Th-based devices have slightly higher saturation current density than the PTB7-based device, consistent with the trend of $J_{\text {sc }}$ values, and is attributed to its broader spectral response (Supplementary Table 5). Under $J_{\text {sc }}$ conditions, the $P(E, T)$ is $94 \%$ for PTB7:hPDI3 and as high as $97 \%$ for PTB7:hPDI4, PTB7Th:hPDI3 and PTB7-Th:hPDI4 devices. From the light-intensity- 
a

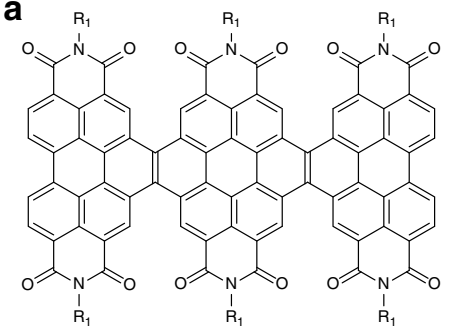

hPDI3<smiles></smiles>

PTB7<smiles></smiles>

$\mathrm{R}_{1}$<smiles></smiles>

PTB7-Th

C

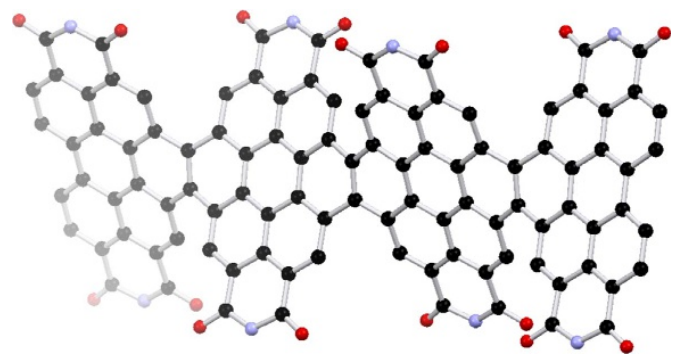

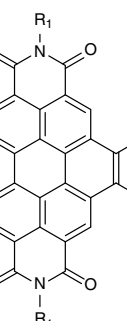

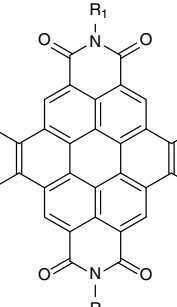

hPDI4

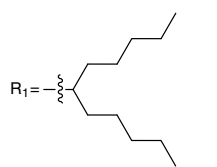

b
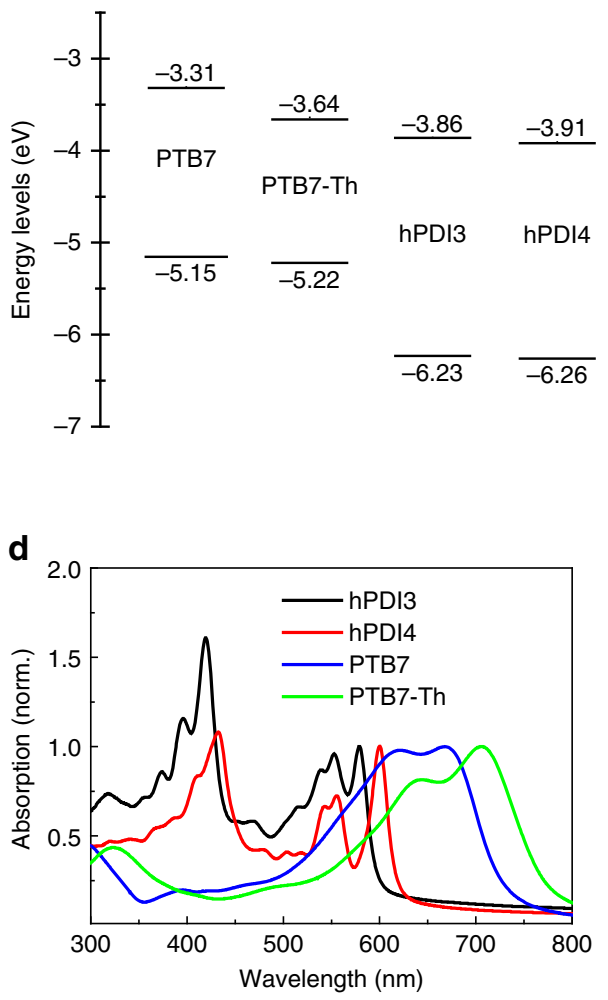

Figure 1 | Molecular structures of helical perylene diimide oligomers and polymers. (a) Chemical structures of hPDI3, hPDI4, PTB7 and PTB7-Th. (b) Schematic of the energy levels of hPDI3, hPDI4, PTB7 and PTB7-Th. Energy levels of PTB7 and PTB7-Th were adopted from ref. 7 and ref. 5, respectively. Energy levels of hPDI3 and hPDI4 were adopted from ref. 21. (c) Molecular model of hPDI4 in a waggling conformation from DFT calculations. Hydrogens and alkyl side chains have been removed for clarity. Black, carbon; red, oxygen; blue, nitrogen. (d) Film absorption spectra of hPDI3, hPDI4, PTB7 and PTB7Th normalized at their low-energy $\lambda_{\max }$.
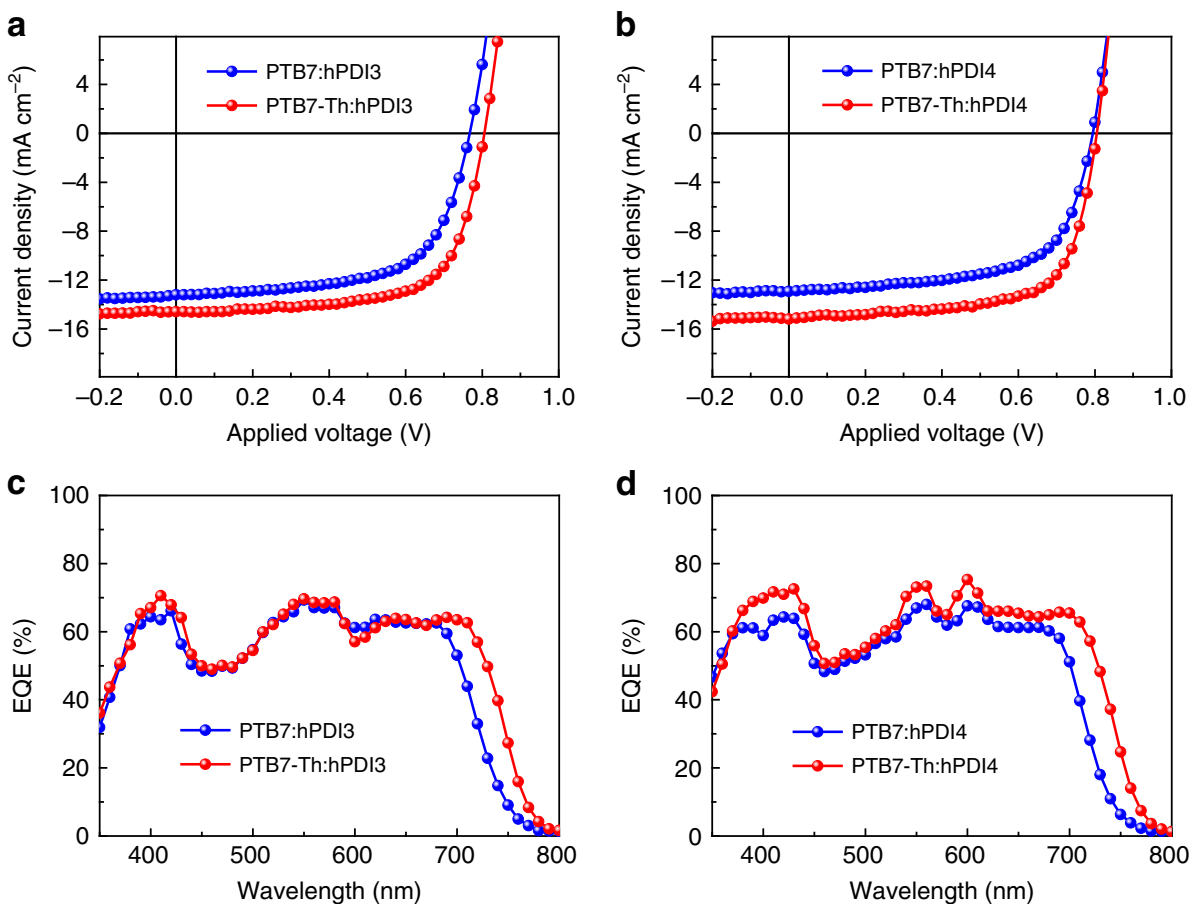

Figure 2 | Device performance of best solar cells based on molecular helices. J-V curves for (a) PTB7:hPDI3 and PTB7-Th:hPDI3 solar cells, (b) PTB7:hPDI4 and PTB7-Th:hPDI4 solar cells under optimized conditions and simulated AM 1.5G irradiation $\left(100 \mathrm{~mW} \mathrm{~cm}^{-2}\right)$. EQE spectra of (c) PTB7:hPDI3, PTB7-Th:hPDI3 and (d) PTB7:hPDI4, PTB7-Th:hPDI4 solar cells under optimized conditions. 
Table 1 | Summary of device parameters of the best solar cells.

\begin{tabular}{lcccc} 
& $\left.\mathbf{J}_{\mathbf{s c}} \mathbf{( m A ~ c m}^{-\mathbf{2}}\right)$ Highest/average & $\boldsymbol{V}_{\mathbf{~ o c}}(\mathbf{V})$ Highest/average & FF (\%) Highest/average & PCE (\%) Highest/average \\
\hline PTB7:hPDI3 & $13.2 / 13.0 \pm 0.2$ & $0.77 / 0.76 \pm 0.01$ & $63 / 62 \pm 1$ & $6.4 / 6.3 \pm 0.1$ \\
PTB7-Th:hPDI3 & $14.5 / 14.3 \pm 0.3$ & $0.81 / 0.80 \pm 0.01$ & $67 / 67 \pm 1$ & $7.9 / 7.7 \pm 0.2$ \\
PTB7:hPDI4 & $12.9 / 12.7 \pm 0.3$ & $0.79 / 0.78 \pm 0.01$ & $64 / 63 \pm 1$ & $6.5 / 6.4 \pm 0.1$ \\
PTB7-Th:hPDI4 & $15.2 / 15.0 \pm 0.2$ & $0.80 / 0.80 \pm 0.01$ & $68 / 68 \pm 1$ & $8.3 / 8.1 \pm 0.2$ \\
PTB7-Th: hPDI4* & 15.1 & 0.802 & 68.2 & 8.27 \\
\hline FF, fill factor; PCE, power conversion efficiency. & & & \\
*Values were measured and certified by Newport Corporation. & & & & \\
\hline
\end{tabular}

dependent current density measurement, we find a near-unity exponent $\alpha$ in the expression of $J_{\mathrm{sc}}$ versus $I^{\alpha}$, where $I$ is incident light intensity (see Supplementary Figs 9-16). This data also suggests that bimolecular recombination and space-charge effect are suppressed at $J_{\text {sc }}$ conditions ${ }^{27}$.

We measure the electron and hole mobilities in the optimal blend films by the space-charge-limited current method ${ }^{28}$. For PTB7-Th:hPDI3 blended film, the hole and electron mobilities are $(1.0 \pm 0.1) \times 10^{-4}$ and $(1.5 \pm 0.1) \times 10^{-4} \mathrm{~cm}^{2} \mathrm{~V}^{-1} \mathrm{~s}^{-1}$, respectively. For PTB7-Th:hPDI4 blended film, the hole and electron mobilities are $(1.2 \pm 0.1) \times 10^{-4}$ and $(1.5 \pm 0.3)$ $\times 10^{-5} \mathrm{~cm}^{2} \mathrm{~V}^{-1} \mathrm{~s}^{-1}$, respectively (see Supplementary Fig. 17 and Supplementary Note 2). The lower electron mobility in PTB7Th:hPDI4 compared with that of PTB7-Th:hPDI3 is probably due to reduced mass ratio of the electron acceptor in the blended film (50\% in PTB7-Th:hPDI4 versus $60 \%$ in PTB7-Th:hPDI3). PTB7Th:hPDI4 blended film exhibits similar electron mobility to fullerene-based blended film ${ }^{29}$, while PTB7-Th:hPDI3 shows even more balanced electron and hole mobility.

Figure 2c,d display the external quantum efficiency (EQE) spectra for each of the devices. These devices show broad and strong photoresponse from 350 to $800 \mathrm{~nm}$. Compared with the PTB7-based solar cells, the PTB7-Th solar cells show a significant increase in the photoresponse between 700 and $800 \mathrm{~nm}$ due to the red-shifted absorption of PTB7-Th. The integrated $J_{\mathrm{sc}}$ values for PTB7:hPDI3, PTB7:hPDI4, PTB7-Th:hPDI3 and PTB7Th:hPDI4 are $13.2,13.1,14.4$ and $15.1 \mathrm{~mA} \mathrm{~cm}^{-2}$, respectively. These values agree well with measured values with a mismatch that is within $2 \%$ for all the devices. Spectra for the solar cells based on hPDI4 show an increase at $600 \mathrm{~nm}$ due to the intense absorption band for hPDI4 in this region. It is notable that all the EQE data consist of maximal transition bands around $550 \mathrm{~nm}$ that is mainly from the absorption of the acceptors. For those PTB7-Th-based solar cells, the highest EQE values are even beyond 70\%. The important conclusion is that the photogenerated excitons from electron acceptors contribute greatly to the photocurrent in these solar cells.

Exciton generation and charge transfer. Two advantages of these molecules over $\mathrm{C}_{60}$ and its derivatives are its significantly higher optical absorption cross-section for the molecules in the visible part of the solar spectrum and its easily tunable bandgap. The helical electron acceptors can thus complement low-bandgap electron donors for an efficient harvesting of solar radiation in a broad wavelength region. To establish this dual light-harvesting mechanism, we use TA spectroscopy to investigate exciton generation in the donor and acceptor, and charge transfer between the two materials.

Figure 3 a compares TA spectra for neat films of hPDI3, PTB7 and the blend at 0 and $5 \mathrm{ps}$, respectively, on photoexcitation at $670 \mathrm{~nm}$. At this wavelength, only PTB7 is excited, as confirmed by the absence of TA signal for hPDI3 (red). The spectrum from neat PTB7 (black) features ground-state bleaching (GB) peaks at 620 and $680 \mathrm{~nm}$, and a broad excited state absorption (ESA) peaks at $\sim 1,400 \mathrm{~nm}$; these features decay bi-exponentially with time constants of $3.2 \pm 0.3$ and $50 \pm 4$ ps (see Supplementary Fig. 18 and Supplementary Note 3). In the blend, this ESA feature decays rapidly, with two time constants of $0.12 \pm 0.03$ and $1.4 \pm 0.2$ ps (Fig. $3 b$ ), suggesting ultrafast electron transfer from photoexcited PTB7 to the electron acceptor, hPDI3. The ultrafast decay of the ESA at $\sim 1,410 \mathrm{~nm}$ is accompanied by a new ESA at $\sim 1,120 \mathrm{~nm}$ that is assigned to hole polaron absorption in PTB7. This assignment is consistent with previous spectroscopic measurement on chemical doped PTB7 that showed a polaron band at the same wavelength ${ }^{30}$. More evidences of this assignment are contained in the Supplementary Figs 19 and 20, and Supplementary Note 4.

The dynamics of exciton dissociation into charge carriers is characterized by two time scales, as shown by the biexponential fit $\left(\tau_{1}=0.12 \pm 0.03 \mathrm{ps}\right.$ and $\left.\tau_{2}=1.4 \pm 0.2 \mathrm{ps}\right)$ to the ESA signal probed at $1,410 \mathrm{~nm}$ (green dots and fit in Fig. $3 \mathrm{~b}$ ). This is more obvious in the ESA signal at $\sim 765 \mathrm{~nm}$ (red triangles in Fig. 3b) that is negligible for the neat PTB7 but appears in the blend. The new ESA in this region is assigned to the polaron absorption both in hPDI3 and PTB7. Bi-exponential fit (red curve, in Fig. 3b) to the dynamics at $765 \mathrm{~nm}$ yields time constants of $\tau_{1}=0.12 \pm 0.03$ ps $\left(70 \%\right.$ relative weight) and $\tau_{2}=1.3 \pm 0.2$ ps (30\% relative weight). These time constants are identical to those obtained from fit-to-signal at $1,410 \mathrm{~nm}$ and both wavelengths probe the same dynamics: the decay of exciton in PTB7 $(1,410 \mathrm{~nm})$ and rise of polaron absorption $(765 \mathrm{~nm})$. We assign $\tau_{1}(0.12 \pm 0.03 \mathrm{ps})$ to the ultrafast exciton dissociation of PTB7 at the interface with electron acceptor hPDI3 (refs 16,31-35) and $\tau_{2}(1.3 \pm 0.2 \mathrm{ps}$ ), to the diffusion of excitons in PTB7 towards interfaces before dissociation $^{36}$. Further confirming the assignment of chargetransfer dynamics, we show that the appearance of GB from hPDI3 at $555 \mathrm{~nm}$ tracks the charge-transfer dynamics (blue squares and fit in Fig. 3b).

We now turn to complementary light harvesting by hPDI3 at its peak absorption wavelength of $415 \mathrm{~nm}$ (see Fig. 1d). For neat hPDI3, excitation at this wavelength leads to GB at 555 and $580 \mathrm{~nm}$, as well as a broad ESA feature in the range of $\sim 600-1,000 \mathrm{~nm}$ (Fig. $3 \mathrm{c}$ ). In the blend, excitation of hPDI3 leads to a rise of the bleaching of PTB7 at $680 \mathrm{~nm}$. This bleaching signal grows with two time constants: $\tau_{1}{ }^{\prime}=0.14 \pm 0.02 \mathrm{ps}$ and $\tau_{2}{ }^{\prime}=1.2 \pm 0.3 \mathrm{ps}$ (green dots and fit in Fig. $3 \mathrm{~d}$ ). The former is assigned to ultrafast hole transfer from photoexcited hPDI3 to PTB7 and the latter to exciton diffusion time in hPDI3 towards interfaces before hole transfer. Exciton dissociation is also evident by the quenching of fluorescence from PTB7 on excitation of hPDI3 (Supplementary Fig. 21 and Supplementary Note 5). This interpretation is supported by the growth of ESA of hole polaron absorption in PTB7 at 1,120 nm (see Fig. 3c, blue spectrum at $5 \mathrm{ps})$. The charge separation process in the blend leads to an approximately two orders of magnitude increase in the time constant for the recovery of hPDI3 (probed at $555 \mathrm{~nm}$, blue triangles in Fig. 3d) as compared with the same process in neat hPDI3 (light blue crosses in Fig. 3d). The similar longer carrier 

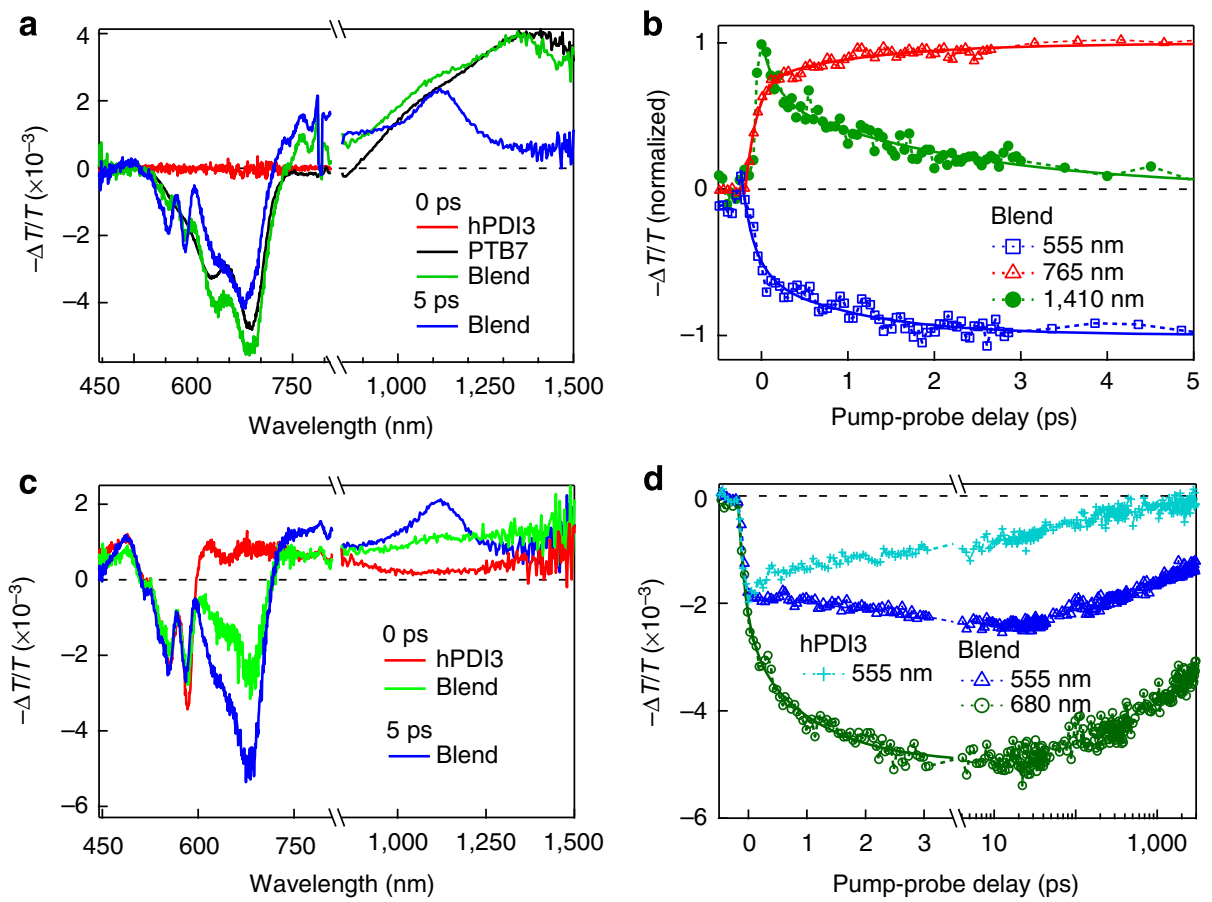

Figure 3 | Spectral and temporal resolved ultrafast exciton dissociation. TA spectra for the films of neat hPDI3, neat PTB7 and their blended films, excited by 670 (a) and $415 \mathrm{~nm}$ (c). (b) Normalized dynamics in the blend at $555 \mathrm{~nm}$ (hPDI3 bleaching peak), polaron absorption at $765 \mathrm{~nm}$ and exciton ESA peak in PTB7 at 1,410 nm, on $670 \mathrm{~nm}$ excitation. (d) Dynamics at $555 \mathrm{~nm}$ for the neat hPDI3 and at 555 and $680 \mathrm{~nm}$ for the blended film on $415 \mathrm{~nm}$ excitation. The curve for neat hPDI3 (light blue) was scaled by a factor of 0.8 . The pump density was $\sim 20 \mu \mathrm{J} \mathrm{cm}{ }^{-2}$ per pulse. Full lines in panels $b$ and $d$ are fits to the data.

lifetime was also found in the blended film at different excitation wavelengths (Supplementary Fig. 18). The much longer carrier recombination times in the blend than those in neat films are attributed to slower bimolecular recombination of the spatially separated charges in the blend.

The TA results presented above establish that photoexcitation of either the donor or the acceptor contribute to charge-carrier generation. Similar to $\mathrm{PC}_{71} \mathrm{BM}$-based $\mathrm{BHJs}{ }^{31-34}$, ultrafast exciton generation from these acceptors in the blended film compensates photoexcitation of the donor, resulting in broad photoresponse over the visible light region. The prevalent use of fullerene has led to the proposal that the spherical shape of fullerene plays a unique role in providing connectivity and electronic delocalization essential for ultrafast charge separation ${ }^{14,37}$. However, our findings show that fullerene is not particularly special and the helical conjugated molecules can also lead to efficient charge separation in BHJs.

Morphology characterization. It is well known that proper phase separation and interpenetrating networks of donor and acceptor domains are critical to charge separation and carrier transport. Many studies on the film morphology in polymer/fullerene solar cells suggest optimal morphology for device operation is to have aggregated domains with sizes on approximately tens of nanometres for both the donor and acceptor ${ }^{13,38}$. However, film morphology of non-fullerene solar cells has received less attention ${ }^{15-18}$. To study the morphology of our blend films, we first performed grazing-incident X-ray diffraction (GIXD) to understand molecular packing in our blend films. Neat films of both PTB7 and PTB7-Th are semicrystalline, as evident by the presence of their (100) reflections located at $q=0.35$ and $q=0.30 \AA^{-1}$, respectively, in the corresponding X-ray diffraction patterns in Supplementary Fig. 22a,b (refs 39,40). The $\mathrm{X}$-ray diffraction patterns also reveal (010) reflections in the out-of-plane direction between $q=1.55$ and $q=1.85 \AA^{-1}$. As the
(010) reflections correspond to the direction of $\pi$-stacking, the intensity anisotropy corresponding to the (010) reflections indicate that both PTB7 and PTB7-Th preferentially adopt a face-on orientation. This assertion is consistent with the observation that the intensities of the (100) reflections of the polymer donors are each concentrated at the equator. The fact that the intensity distributions associated with the (100) and (010) reflections of PTB7-Th are sharper than those associated with the corresponding reflections of PTB7 indicates that the crystallites of PTB7-Th exhibit a higher degree of face-on orientation compared with PTB7. The GIXD pattern of hPDI3 in Supplementary Fig. 22c also exhibits a first-order reflection at $q=0.35 \AA^{-1}$. Given that the intensity distribution of this reflection does not vary substantially with azimuthal angle, crystallites of hPDI3 do not adopt any preferential orientation. Supplementary Fig. 22d,e exhibit the GIXD patterns of thin films comprising each of the polymer donor with hPDI3. The primary reflections of the polymer donors and hPDI3 overlap substantially. We are thus not able to make meaningful comparison based on the intensities in this $q$-range. Comparing the GIXD patterns of the blends at higher $q$-range with those of the constituent polymer donors does, however, reveal differences. We observe the (010) reflections in the GIXD patterns of the blends to be substantially weaker than those in the patterns of the polymer donor constituents. Further, the weak intensities associated with the (010) reflections in the GIXD patterns of the blends appear to be more isotropic with azimuthal angle. Collectively, these observations suggest the addition of hPDI3 to disrupt the crystallization of the polymer donor constituents and crystallites that form are less oriented compared with those in thin films comprising the polymer donor constituents alone.

We investigated the PTB7-Th:hPDI3-blended film morphology using both tapping mode and quantitative nanomechanical mode $^{41}$ atomic force microscopy (AFM) (Fig. 4). Although the height image of the top film surface is very smooth (root-mean- 

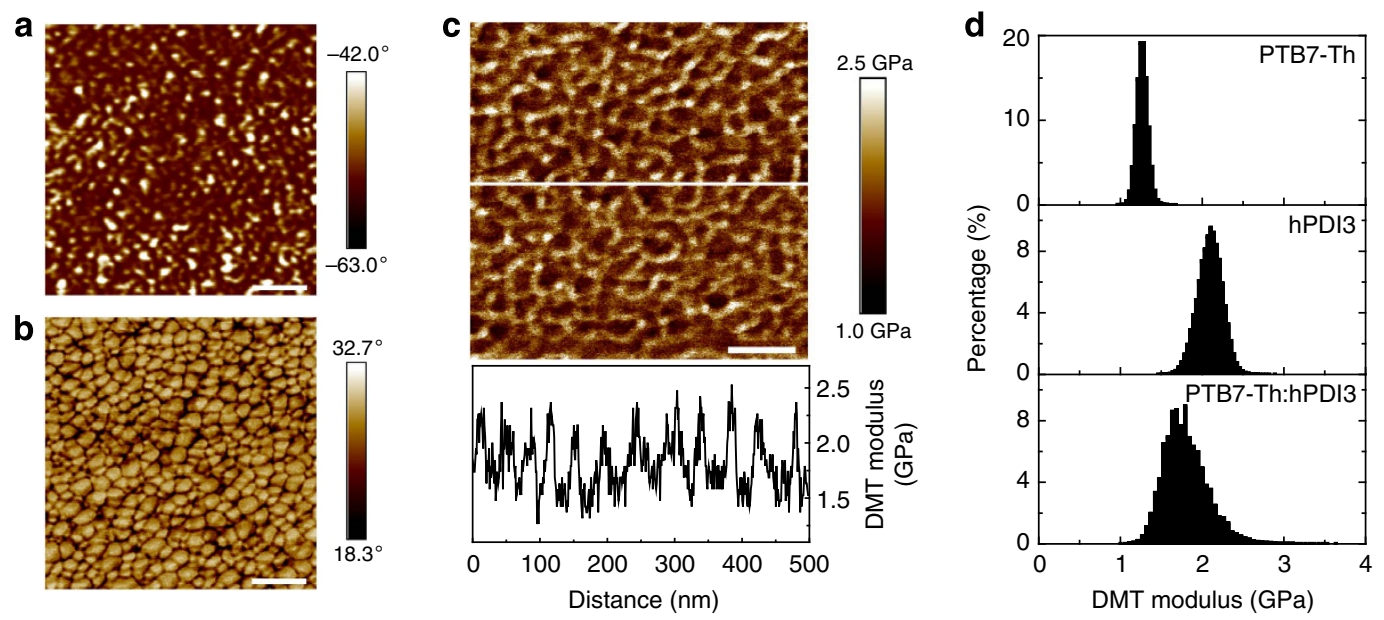

Figure 4 | Film morphology of PTB7-Th: hPDI3 blend film. (a) Top surface phase image of BHJ thin film measured in tapping mode. (b) Internal phase image of blended thin film measured in tapping mode. (c) Internal DMT (Derjaguin, Muller, Toropov) modulus image of blended thin film measured in peak force quantitative nanomechanical (QNM) mode. Bottom graph is line-cut analysis of image. (c,d) DMT modulus of PTB7-Th and hPDI3 pure thin films and PTB7-Th:hPDI3 blend film. Scale bar, $100 \mathrm{~nm}(\mathbf{a}-\mathbf{c})$.

squared (RMS) roughness of $0.58 \mathrm{~nm}$ ), the phase image shows evidence of a distinct phase separation, with domain size of $\sim 10$ $20 \mathrm{~nm}$ (Fig. 4a and Supplementary Fig. 23). We used an oxygen plasma to remove $\sim 30 \mathrm{~nm}$ of material from the top surface to investigate the blend's internal morphology ${ }^{41-43}$. Here, the phase image clearly shows a continuous interpenetrating network with a feature size in the range of $20-40 \mathrm{~nm}$ (Fig. 4b) - a morphology clearly favourable for exciton dissociation and charge transport. Nanomechanical measurements show that the continuous network (dark regions in Fig. 4b) has a DMT (Derjaguin, Muller, Toropov) modulus of $\sim 2.2 \mathrm{GPa}$, which is similar to that of the pure film of hPDI3 (details about DMT model in Supplementary Note 6). However, the isolated embedded in the continuous network domains (bright regions in Fig. 4b) have a smaller DMT modulus $(\sim 1.5 \mathrm{GPa})$, closer to that of a pure PTB7-Th film (Fig. 4c,d). These results suggest an active layer composed of an interpenetrating network of hPDI3-rich domains, embedded in PTB7-Th-rich matrix. Our blend films share very similar morphology to that of $\mathrm{PTB} 7: \mathrm{PC}_{71} \mathrm{BM}$, which is considered an optimal morphology to enable efficient charge generation and transport in BHJ solar cells. ${ }^{41}$

\section{Conclusion}

This study introduces a new class of highly efficient electron acceptors for organic BHJ solar cells, consisting of helical conjugated PDI oligomers. We have demonstrated solar cells with power conversion efficiencies comparable to previous reports from devices using fullerene derivatives. We believe the values reported here represent a lower limit for what is achievable, and that creating electron donor materials that are matched both structurally and electronically with these molecules is a clear path to further improvement.

\section{Methods}

Materials. hPDI3 and hPDI4 were synthesized according to a previously reported method $^{20}$. PTB7 and PTB7-Th were purchased from 1-materal Inc. Synthesis of $\mathrm{ZnO}$ sol-gel precursor was described elsewhere ${ }^{44}$. Zinc acetate dihydrate, ethanolamine, 2-methoxyethanol, DIO and all of the solvents were purchased from Sigma Aldrich.

Device fabrication. Prepatterned ITO-coated glass with a sheet resistance of $\sim 15 \Omega \mathrm{sq}^{-1}$ was cleaned with detergent and ultrasonicated in deionized water, acetone and isopropanol for $30 \mathrm{~min}$, respectively. Subsequently, we treated the substrates by ultraviolet-ozone for $10 \mathrm{~min}$. The prepared $\mathrm{ZnO}$ precursor was spin-cast onto the ITO substrate at 3,000 r.p.m. for $1 \mathrm{~min}$, followed by annealing at $200^{\circ} \mathrm{C}$ for $1 \mathrm{~h}$ in air, to form a thin film with approximate thickness of $20 \mathrm{~nm}$. Active layers were prepared by spin-coating a mixed solution containing polymer and acceptor in chlorobenzene at a total concentration of $25 \mathrm{mg} \mathrm{ml}^{-1}$. The thickness of the prepared active layers is about $100 \mathrm{~nm}$. Active layers were heated at $80^{\circ} \mathrm{C}$ for $10 \mathrm{~min}$ in the nitrogen filled glove box to remove the residual solvent. Finally, a 7-nm $\mathrm{MoO}_{3}$ layer was deposited first and then a 100-nm Al electrode were subsequently deposited through a shadow mask by thermal evaporation under a vacuum about $1 \times 10^{-6}$ torr.

Characterization. The current density-voltage $(J-V)$ curves were measured by a Keithley 2635A source measure unit. The photocurrent was measured under AM $1.5 \mathrm{G}$ illumination at $100 \mathrm{~mW} \mathrm{~cm}^{-2}$ under a Newport solar Simulator. A KG5-Si reference cell traceable to Newport was used to calibrate light intensity. Mismatch between integrated $J_{\mathrm{sc}}$ values from EQE with the AM 1.5G solar spectrum and the measured values is within $2 \%$. Spectral mismatch factors $(M)$ calculated according to a standard procedure reported elsewhere ${ }^{45}$ were applied to all the devices. The effective device area was defined as $6.25 \mathrm{~mm}^{2}$ by an aperture mask, except that a $4-\mathrm{mm}^{2}$ aperture mask was used for the certified device at Newport Corporation. Average device parameters were obtained from at least 6 devices at each condition and at least 12 devices at the optimal condition for each donor-acceptor combination. EQE measurements were performed using a QE system from PV Measurements Inc. Absorption spectra were obtained on Shimadzu UV 1800 ultraviolet-visible.

TA spectroscopy. To investigate charge transfer in the solar cells, we employed the TA spectroscopy. The pump laser light ( $\sim 100 \mathrm{fs}$ pulse width) comes from an optical parametric amplifier pumped by a Ti:sapphire femtosecond regenerative amplifier $(800 \mathrm{~nm}, 1 \mathrm{kHz}$ rep-rate). The probe light is a white-light supercontinuum (450-900 and 850-1,600 nm for the visible and near-infrared range, respectively). The pump and probe beams overlapped under a small angle. The detection consists of a pair of high-resolution multichannel detector arrays coupled to a high-speed data acquisition system.

Grazing-incident X-ray diffraction. GIXD experiments were conducted at the G1 station $(11.8 \pm 0.1 \mathrm{keV})$ of the Cornell High Energy Synchrotron Source. The beam was chosen to be $0.05 \mathrm{~mm}$ tall and $1 \mathrm{~mm}$ wide. The width of each sample was $5 \mathrm{~mm}$. The X-ray beam was aligned above the film's critical angle but below that of the substrate, at a $0.18^{\circ}$ incident angle with the substrate. X-ray scattering was collected with a two-dimensional Pilatus $200 \mathrm{~K}$ detector, positioned $84.5 \mathrm{~mm}$ from the sample. All GIXD images have been background subtracted.

Atomic force microscopy. AFM measurements were carried out in tapping mode and PeakForce quantitative nanomechanical mode on a Bruker Multi-Mode AFM at ambient conditions. A commercial silicon cantilever (RTESPA, MPP-11120-10, Bruker) was used in this study with a typical radius of curvature of $\sim 8 \mathrm{~nm}$ and a nominal spring constant of $\sim 40 \mathrm{Nm}^{-1}$. Nanomechanical mapping was operated at constant peak force. The etching treatment was performed on the sample surface 
using oxygen plasma (Plasma Etch Inc., Model: PE-50) to etch out $\sim 30 \mathrm{~nm}$ from the top surface.

\section{References}

1. Liu, Y. et al. Aggregation and morphology control enables multiple cases of high-efficiency polymer solar cells. Nat. Commun. 5, 5293 (2014).

2. Liao, S.-H. et al. Single junction inverted polymer solar cell reaching power conversion efficiency $10.31 \%$ by employing dual-doped zinc oxide nano-film as cathode interlayer. Sci. Rep. 4, 6813 (2014).

3. Heliatek consolidates its technology leadership by establishing a new world record for organic solar technology with a cell efficiency of $12 \%$. Available at http://www.heliatek.com/en/press/press-releases/details/heliatek-consolidatesits-technology-leadership-by-establishing-a-new-world-record-for-organicsolar-technology-with-a-cell-effi (2013).

4. Liang, Y. et al. For the bright future-bulk heterojunction polymer solar cells with power conversion efficiency of 7.4\%. Adv. Mater. 22, E135-E138 (2010).

5. Liao, S.-H., Jhuo, H.-J., Cheng, Y.-S. \& Chen, S. -A. Fullerene derivative-doped zinc oxide nanofilm as the cathode of inverted polymer solar cells with low-bandgap polymer (PTB7-Th) for high performance. Adv. Mater. 25, 4766-4771 (2013).

6. Lee, J. K. et al. Processing additives for improved efficiency from bulk heterojunction solar cells. J. Am. Chem. Soc. 130, 3619-3623 (2008).

7. He, Z. C. et al. Enhanced power-conversion efficiency in polymer solar cells using an inverted device structure. Nat. Photon. 6, 591-595 (2012).

8. Page, Z. A., Liu, Y., Duzhko, V. V., Russell, T. P. \& Emrick, T. Fulleropyrrolidine interlayers: tailoring electrodes to raise organic solar cell efficiency. Science 346, 441-444 (2014).

9. Zhang, Z.-G. et al. Perylene diimides: a thickness-insensitive cathode interlayer for high performance polymer solar cells. Energy Environ. Sci. 7, 1966-1973 (2014).

10. You, J. et al. A polymer tandem solar cell with $10.6 \%$ power conversion efficiency. Nat. Commun. 4, 1446 (2013).

11. Lu, L., Xu, T., Chen, W., Landry, E. S. \& Yu, L. Ternary blend polymer solar cells with enhanced power conversion efficiency. Nat. Photon. 8, 716-722 (2014).

12. Cnops, K. et al. $8.4 \%$ efficient fullerene-free organic solar cells exploiting long-range exciton energy transfer. Nat. Commun. 5, 3406 (2014)

13. Dou, L. et al. 25th Anniversary article: a decade of organic/polymeric photovoltaic research. Adv. Mater. 25, 6642-6671 (2013).

14. Savoie, B. M. et al. Unequal partnership: asymmetric roles of polymeric donor and fullerene acceptor in generating free charge. J. Am. Chem. Soc. 136, 2876-2884 (2014)

15. Zang, Y. et al. Integrated molecular, interfacial, and device engineering towards high-performance non-fullerene based organic solar cells. Adv. Mater. 26, 5708-5714 (2014)

16. Zhong, Y. et al. Efficient organic solar cells with helical perylene diimide electron acceptors. J. Am. Chem. Soc. 136, 15215-15221 (2014).

17. Lin, Y. et al. An electron acceptor challenging fullerenes for efficient polymer solar cells. Adv. Mater. 27, 1170-1174 (2015).

18. Lin, Y. et al. High-performance fullerene-free polymer solar cells with $6.31 \%$ efficiency. Energy Environ. Sci. 8, 610-616 (2015).

19. Jung, J. W. et al. Fluoro-substituted n-type conjugated polymers for additivefree all-polymer bulk heterojunction solar cells with high power conversion efficiency of 6.71\%. Adv. Mater. 27, 3310-3317 (2015).

20. Zhong, Y. et al. Helical ribbons for molecular electronics. J. Am. Chem. Soc. 136, 8122-8130 (2014).

21. Hiszpanski, A. M. et al. Halogenation of a nonplanar molecular semiconductor to tune energy levels and bandgaps for electron transport. Chem. Mater. 27, 1892-1900 (2015).

22. Li, H. et al. Beyond fullerenes: design of nonfullerene acceptors for efficient organic photovoltaics. J. Am. Chem. Soc. 136, 14589-14597 (2014).

23. Ball, M. et al. Contorted polycyclic aromatics. Acc. Chem. Res. 48, 267-276 (2015).

24. Savoie, B. M. et al. Mesoscale molecular network formation in amorphous organic materials. Proc. Natl Acad. Sci. USA 111, 10055-10060 (2014)

25. Chen, J.-D. et al. Single-junction polymer solar cells exceeding $10 \%$ power conversion efficiency. Adv. Mater. 27, 1035-1041 (2014).

26. Cui, C., Wong, W.-Y. \& Li, Y. Improvement of open-circuit voltage and photovoltaic properties of 2D-conjugated polymers by alkylthio substitution. Energy Environ. Sci. 7, 2276-2284 (2014).

27. Cowan, S.vR., Roy, A. \& Heeger, A.vJ. Recombination in polymer-fullerene bulk heterojunction solar cells. Phys. Rev. B 82, 245207 (2010)

28. Malliaras, G. G., Salem, J. R., Brock, P. J. \& Scott, C. Electrical characteristics and efficiency of single-layer organic light-emitting diodes. Phys. Rev. B 58, R13411-R13414 (1998).

29. Lee, B. R. et al. Amine-based polar solvent treatment for highly efficient inverted polymer solar cells. Adv. Mater. 26, 494-500 (2014).
30. Basel, T. et al. Optical, electrical, and magnetic studies of organic solar cells based on low bandgap copolymer with spin $1 / 2$ radical additives. Adv. Funct. Mater. 25, 1895-1902 (2015).

31. Provencher, F. et al. Direct observation of ultrafast long-range charge separation at polymer-fullerene heterojunctions. Nat. Commun. 5, 4288 (2014)

32. Gélinas, S. et al. Ultrafast long-range charge separation in organic semiconductor photovoltaic diodes. Science 343, 512-516 (2014).

33. Bernardo, B. et al. Delocalization and dielectric screening of charge transfer states in organic photovoltaic cells. Nat. Commun. 5, 3245 (2014).

34. Tamura, H. \& Burghardt, I. ultrafast charge separation in organic photovoltaics enhanced by charge delocalization and vibronically hot exciton dissociation. J. Am. Chem. Soc. 135, 16364-16367 (2013).

35. Jailaubekov, A. E. et al. Hot charge-transfer excitons set the time limit for charge separation at donor/acceptor interfaces in organic photovoltaics. Nat. Mater. 12, 66-73 (2013).

36. Kaake, L. G. et al. Photoinduced charge generation in a molecular bulk heterojunction material. J. Am. Chem. Soc. 134, 19828-19838 (2012).

37. Savoie, B. M., Jackson, N. E., Chen, L. X., Marks, T. J. \& Ratner, M. A. Mesoscopic features of charge generation in organic semiconductors. Acc. Chem. Res. 47, 3385-3394 (2014).

38. Chen, W. et al. Hierarchical nanomorphologies promote exciton dissociation in polymer/fullerene bulk heterojunction solar cells. Nano Lett. 11, 3707-3713 (2011).

39. Hammond, M. R. et al. Molecular order in high-efficiency polymer/fullerene bulk heterojunction solar cells. ACS Nano 5, 8248-8257 (2011).

40. Huang, W. et al. Unraveling the morphology of high efficiency polymer solar cells based on the donor polymer PBDTTT-EFT. Adv. Energy Mater. 5, 1401259 (2015)

41. Wang, D. et al. New insights into morphology of high performance BHJ photovoltaics revealed by high resolution AFM. Nano Lett. 14, 5727-5732 (2014).

42. Hedley, G. J. et al. Determining the optimum morphology in high-performance polymer-fullerene organic photovoltaic cells. Nat. Commun. 4, 2867 (2013).

43. Kong, J., Hwang, I.-W. \& Lee, K. Top-down approach for nanophase reconstruction in bulk heterojunction solar cells. Adv. Mater. 26, 6275-6283 (2014).

44. Jo, J. et al. Enhanced efficiency of single and tandem organic solar cells incorporating a diketopyrrolopyrrole-based low-bandgap polymer by utilizing combined $\mathrm{ZnO} /$ polyelectrolyte electron-transport layers. Adv. Mater. 25, 4783-4788 (2013).

45. Shrotriya, V. et al. Accurate measurement and characterization of organic solar cells. Adv. Funct. Materials 16, 2016-2023 (2006).

\section{Acknowledgements}

Primary support for this project was provided by the Chemical Sciences, Geosciences and Biosciences Division, Office of Basic Energy Sciences, US Department of Energy (DOE) under award number DE-FG02-01ER15264. X.Y.Z. acknowledges support by the US National Science Foundation grant DMR 1321405. This research used resources of the Center for Functional Nanomaterials, which is a US DOE Office of Science User Facility, at Brookhaven National Laboratory under contract number DE-SC0012704. Portions of this work was conducted at the Cornell High Energy Synchrotron Source (CHESS), which is supported by the National Science Foundation and the National Institutes of Health/National Institute of General Medical Sciences under NSF awards DMR-0936384 and DMR-1332208. G.E.P. acknowledges Government support under and awarded by DoD, Air Force Office of Scientific Research, National Defense Science and Engineering Graduate (NDSEG) Fellowship, 32 CFR 168a. We thank Columbia University's Shared Materials Characterization Lab for use of the equipment essential to the research.

\section{Author contributions}

Y.Z., F.N., X.-Y.Z. and C.N. conceived the idea. Y.Z. and M.T.T. designed the experiments. Y.Z. performed device fabrication, characterization, AFM experiments and data analysis. M.T.T. conducted TA measurements and data analysis. R.C., W.W. and B.Z. helped with device fabrication and characterization. G.E.P., P.P.K. and M.S performed GIXD measurements and analysed the data. S.O., B.F. and F.N. synthesized and purified compound hPDI3 and hPDI4. H.Z. conducted ultrafast fluorescence upconversion experiments and analysed the data. M.L.S. performed DFT calculations on compound hPDI3 and hPDI4. M.Y.S. helped with experimental setup for TA measurements. Y.Z., M.T.T., G.E.P., X.-Y.Z. and C.N. wrote the manuscript. C.Y.N., C.B., M.L.S. and Y.L.L. contributed to revision of the manuscript. All authors discussed the results and reviewed the manuscript.

\section{Additional information}

Supplementary Information accompanies this paper at http://www.nature.com/ naturecommunications

Competing financial interests: The authors declare no competing financial interests. 
Reprints and permission information is available online at http://npg.nature.com/ reprintsandpermissions/

How to cite this article: Zhong, Y. et al. Molecular helices as electron acceptors in high-performance bulk heterojunction solar cells. Nat. Commun. 6:8242 doi: $10.1038 /$ ncomms9242 (2015). (c) (i) This work is licensed under a Creative Commons Attribution 4.0 International License. The images or other third party material in this article are included in the article's Creative Commons license, unless indicated otherwise in the credit line; if the material is not included under the Creative Commons license, users will need to obtain permission from the license holder to reproduce the material. To view a copy of this license, visit http://creativecommons.org/licenses/by/4.0/ 